The experimental observation of the surfaceopalescence of water presents special difficulties owing to the great ease with which this liquid catches dust and grease. The difficulties have, however, been successfully overcome and the effect satisfactorily demonstrated, both with water rendered dust-free in sealed bulbs and with the water-film on a clean block of melting ice kept free of dust by a gentle stream of gas blowing upon it.

2 ro Bowbazaar Street, Calcutta,

C. V. Raman. June 28.

On Continuous Radiation from the Sun.

Prof. J. Q. STEwart recently published in these columns (NATURE, February Io, p. I86) a very interesting communication on the optical and electrical properties of ionised gases. For some time past I have been engaged in investigations on similar lines, and I wish to direct attention to one important side-result. It is well known that in estimating the surface temperature of heavenly bodies (as has been done by Coblentz, Abbot, Wilsing and Scheiner, and others), from their continuous spectra, it is always tacitly assumed that they radiate like perfectly black bodies. Several investigators have pointed out that this assumption does not tally with experimental results. The temperature obtained by applying the total radiation law and the method of isochromatics to the spectral-energy curve are at variance with each other. They are also different from the temperatures obtained from the ionisation-theory.

The best example is afforded by the sun, which, according to the careful measurements of Abbot and Wilsing, shows a spectral-energy curve considerably deviating from that of a black body (see E. A. Milne, Phil. Trans. vol. 223, p. 2x8); the fact has been discussed by many investigators, including Schwarzschild, Groot, Milne, Dietzius, and others. There are very weighty reasons why the sun would not radiate like a black body. A black body or a full radiator is one which absorbs all the radiant energy which falls on it, reflecting none. Such an ideal body is nowhere met with in the world, but Wien and Lummer realised it by making use of a hollow enclosure maintained at a constant temperature, and provided with a small hole, the idea being that a beam of radiation within the enclosure would describe an infinitely circuitous path, and what the emission lacks in fullness will be made up by repeated reflections.

It is clear that none of these conditions is fulfilled in the case of the sun. The surface of the sun contains a large percentage of free electrons, and positive charges, which endow it with a large reflecting power. This point will be clear if we remember the analogous case of metals. According to the electromagnetic theory, metals derive their high reflecting power from the presence in them of a large number of free electrons, or rather electrons which are easily excited to vibration by incident radiation. A theory of emissivity of metals on this basis was worked out by Aschkinass in 1905, and has been verified by the experiments of Rubens and Hagen, Langmuir, and others.

The presence of a large percentage of free electrons on the surface of the sun would, thus, endow it with a high reflecting power. The surface being an open one, the hollow enclosure condition is not realised. Thus the conclusion seems to be irresistible that the total emission from the surface would fall far short of that of full radiator. The form of the spectral. energy curve suggests the emissivity $E_{\lambda}$ varies as $\frac{\mathrm{A}}{\lambda^{5}+x} \phi(\lambda \theta)$ where $\mathrm{I}>x \geq \frac{1}{2}$, but about this point judgment should be reserved now.

Turning to the stars, it is easy to see that similar conditions would hold. The analogy with metals enables us to say that the emission from low temperature stars would fall far short of that from a full radiator at the same temperature, while for stars with very high temperature, emissivity may approach that of a black body.

Prof. Eddington's work on the constitution of stars is based on the assumption that inside the stars total emissivity varies as $\mathrm{T}^{4}$; this assumption is probably not affected, for, inside the stars, the hollow enclosure condition is largely fulfilled.

University College of Science, Calcutta,

$$
\text { MEgh NAD SAHA. }
$$
July 5 .

\section{Separation of Common Lead into Fractions of Different Density.}

By fractional crystallisation of lead assay foil, about 300 grams in all, two end fractions, each weighing about 60 grams, were obtained. These fractions were then purified according to Stas's method. For the density determinations, about ten grams of each was melted in an atmosphere of hydrogen and allowed to solidify in a vacuum. The densities of samples prepared in this way were determined in specific gravity bottles.

Density of lead from crystals end of fractionating series :- II.345士0.005.

Density of lead from mother liquor end of fractionating series :- I I.3I $3 \pm 0 \cdot 005$.

A sample of Stas lead, which Mr. C. T. Heycock very kindly gave me, was found to have the density II.328 in one experiment and II.326 after re-melting.

The difference in density between the above-mentioned fractions persisted after granulating the metal and also after re-melting the granulated metal under potassium cyanide. It was discovered in the course of these experiments that lead which has solidified slowly is not homogeneous as regards density,- - the parts which freeze first being denser.

Out of eleven experiments, only one was inconsistent with the view that the original lead had been separated into two fractions which had different densities.

The work is being continued.

R. H. AtKinson,

Goldsmiths' Metallurgical Laboratory,

University Chemical Laboratory, Cambridge, July 18 .

\section{Proposed International Survey of the Sky.}

ON the initiative of the French National Meteorological Service, it has been decided to take photographs of the clouds three times daily during the week September 17-23, inclusive, at as many stations as possible throughout the countries of western Europe. As the number of official meteorological stations is limited, it has been proposed to enlist the services of those professional and amateur photographers who are willing to co-operate voluntarily in the work. The photographs should be taken as nearly as possible at 7 A.M., I P.M., and 6 P.M. G.M.T. (not summer time). The photographer should make a note of the direction in which the camera is pointing when the photograph is taken (e.g. north, south-west, etc.); if more than one photograph is taken at any NO. 2808 , vOL. 1 I 2$]$ 